\title{
Lipid engineering reveals regulatory roles for membrane fluidity in yeast flocculation and oxygen-limited growth
}

\author{
Daniel Degreif $^{\mathrm{a}, \mathrm{b}}$, Tristan de Rond ${ }^{\mathrm{a}, \mathrm{c}}$, Adam Bertl ${ }^{\mathrm{b}}$, Jay D. Keasling ${ }^{\mathrm{a}, \mathrm{c}, \mathrm{d}, \mathrm{e}, \mathrm{f}, \mathrm{g}, \mathrm{h}}$, Itay Budin ${ }^{\mathrm{a}, \mathrm{d}, \mathrm{f}, *}$ \\ a Joint BioEnergy Institute, 5885 Hollis Street, Emeryville, CA 94608, USA \\ b Department of Biology, Technische Universität Darmstadt, Darmstadt, Germany \\ c Department of Chemistry, University of California, Berkeley, Berkeley, CA 94720, USA \\ d Department of Chemical \& Biomolecular Engineering, University of California, Berkeley, Berkeley CA 94720, USA \\ e Department of Bioengineering, University of California, Berkeley, Berkeley CA 94720, USA \\ ${ }^{f}$ QB3 Institute, University of California, Berkeley, Berkeley, CA 94270, USA \\ g Biological Systems \& Engineering, Lawrence Berkeley National Laboratory, Berkeley, CA 94720, USA \\ h The Novo Nordisk Foundation Center for Sustainability, Technical University of Denmark, Denmark
}

A R T I C L E I N F O

Keywords:

Fatty acid unsaturation

Membrane fluidity

Yeast flocculation

Oxygen-limited growth

Hypoxia

Fermentation

\begin{abstract}
A B S T R A C T
Cells modulate lipid metabolism in order to maintain membrane homeostasis. Here we use a metabolic engineering approach to manipulate the stoichiometry of fatty acid unsaturation, a regulator of cell membrane fluidity, in Saccharomyces cerevisiae. Unexpectedly, reduced lipid unsaturation triggered cell-cell adhesion (flocculation), a phenomenon characteristic of industrial yeast but uncommon in laboratory strains. We find that ER lipid saturation sensors induce expression of FLO1 - encoding a cell wall polysaccharide binding protein - independently of its canonical regulator. In wild-type cells, Flo1p-dependent flocculation occurs under oxygen-limited growth, which reduces unsaturated lipid synthesis and thus serves as the environmental trigger for flocculation. Transcriptional analysis shows that FLO1 is one of the most highly induced genes in response to changes in lipid unsaturation, and that the set of membrane fluidity-sensitive genes is globally activated as part of the cell's long-term response to hypoxia during fermentation. Our results show how the lipid homeostasis machinery of budding yeast is adapted to carry out a broad response to an environmental stimulus important in biotechnology.
\end{abstract}

\section{Introduction}

The lipid composition of cellular compartments is thought to control the physicochemical properties of their membranes and thus could act as a broad regulator of membrane-localized cellular machinery. Lipid composition can vary tremendously between cells, organelles, and tissues, potentially reflecting differing constraints that molecular processes hosted by these membranes place (van Meer et al., 2008). For example, in budding yeast (Saccharomyces cerevisiae), global lipid analysis by mass spectrometry has shown that the cellular lipidome changes during growth and is dependent on carbon source (Klose et al., 2012). However, understanding functional roles for differences in lipid composition remains a challenge because a limited set of tools is available for studying lipid composition in vivo. Standard genetic approaches, e.g. gene knockouts, provide little functional information on essential lipid components, while chemical tools, such as media supplements (Bossie and Martin, 1989) and lipid chelators
(Zidovetzki and Levitan, 2007), provide limited stoichiometric control over lipid composition.

An alternative strategy to chemical manipulation of lipid composition is to engineer cells in which the expression of lipid synthesis pathways is placed under experimental control. We sought to use this approach to investigate a central chemical parameter in determining the physical state of cell membranes: the proportion of fluidizing double bonds in phospholipid acyl chains (lipid unsaturation). Enzymatic desaturation generates cis-double bonds in acyl chains, whose geometry interferes with inter-lipid interactions, thereby reducing lipid packing and fluidizing the bilayer (Spector and Yorek, 1985). Membrane fluidity is thought to be maintained by cells in response to changes in temperature (Hazel, 1995) or solvents (Ingram, 1976) by modulating the fatty acid composition of membrane phospholipids. Changes in fatty acid unsaturation are also associated with human diseases, including the development of type-2 diabetes (Weijers, 2012) and tumor proliferation in cancer (Igal, 2010). However, the functional

\footnotetext{
* Corresponding author at: Joint BioEnergy Institute, 5885 Hollis Street, Emeryville, CA 94608, USA

E-mail addresses: budin@berkeley.edu, ibudin@lbl.gov (I. Budin).
} 
B

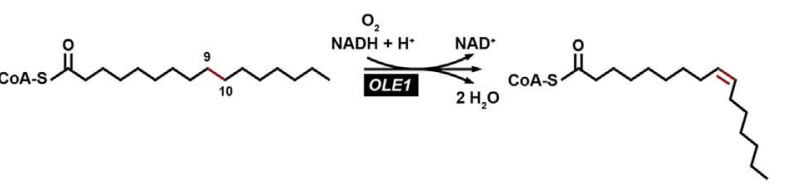

D
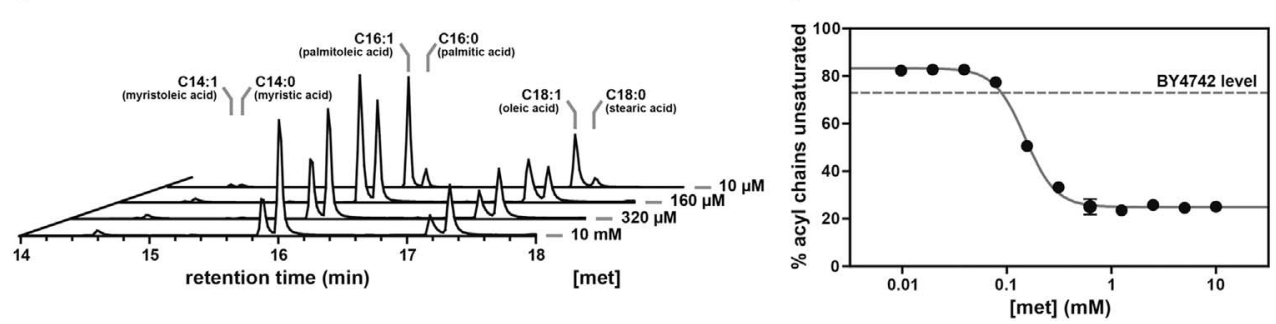
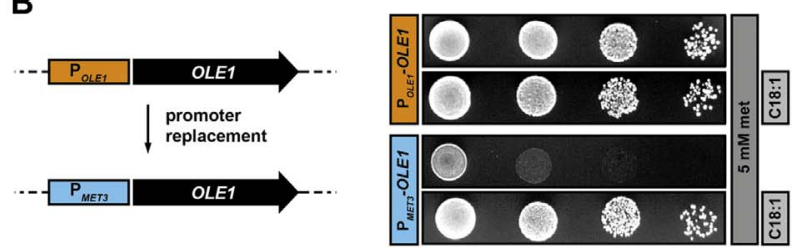

E

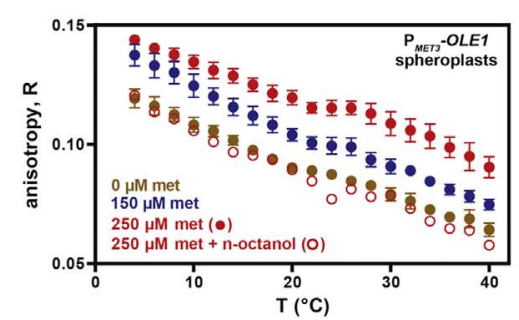

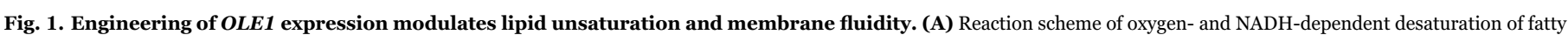

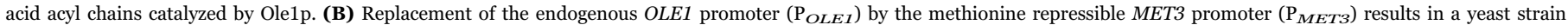

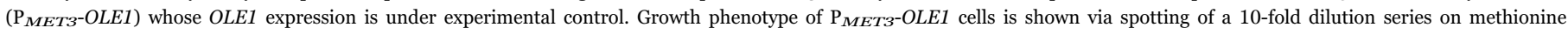

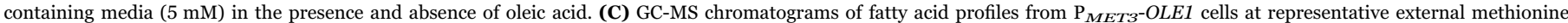

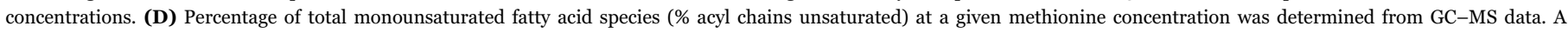

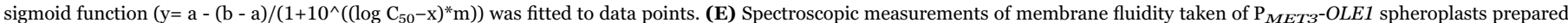

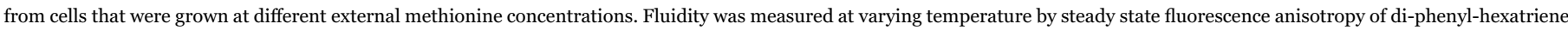

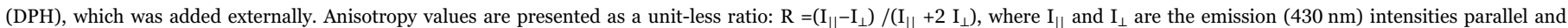

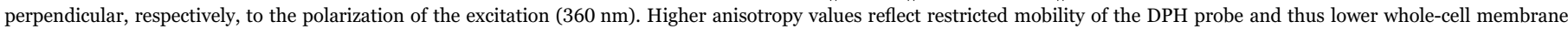

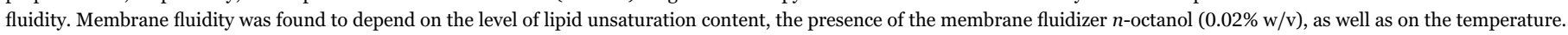
Values in the presence of $n$-octanol are shown as an average of two biological replicates. Error bars, SEM $(n=3)$.

effects of these physical and chemical parameters on basic cellular physiology are still not fully understood. Higher eukaryotes feature a repertoire of desaturases with varying substrates and products (Hashimoto et al., 2008), including polyunsaturated species whose complex biophysical effects make identifying fluidity-regulated functions a challenge (Barelli and Antonny, 2016). In contrast, budding yeast features only a single lipid desaturase (Ole1p), which converts a wide range of coenzyme A-bound fatty acids into their corresponding $\Delta$ 9-cis monounsaturated species (Stukey et al., 1989) (Fig. 1A).

Because bilayer fluidity is an important parameter for membraneassociated cellular processes, yeast has evolved mechanisms for modulating the level of $O L E 1$ expression in response to metabolic stages during growth (Casanovas et al., 2015) and to environmental stimuli, such as the supply of exogenous lipids. In budding yeast, a fatty acid regulatory (FAR) region in the $O L E 1$ promoter was first identified to be responsive to fatty acid metabolism genes (Choi et al., 1996). Later it was found that a pair of ER membrane-bound transcriptional activators - Spt23p and Mga2p (Hoppe et al., 2000), which were first identified as regulators of $T Y$ retrotransposon elements (Zhang et al., 1997) - mediate OLE1-specific lipid regulation. Biochemical and genetic experiments have led to a model in which membrane-anchored Spt23p and Mga2p are proteolytically processed in response to changes in fatty acid composition (Rape et al., 2001). The resulting active fragments, termed p90 domains, induce OLE1 expression, though neither protein contains a recognizable DNA binding domain (Zhang et al., 1997; Burkett and Garfinkel, 1994; Dula and Holmes, 2000). Recent work has proposed a model in which proteolysis is driven by conformational changes in the transmembrane helices of Spt23p/ Mga2p homodimers mediated by the unsaturated lipid content of the ER membrane (Covino et al., 2016). Several key aspects of this pathway are still under investigation, including the exact mechanism by which Spt23p/Mga2p or their interacting partners influence gene expression.

In this study we sought to systematically investigate the physiological effects of changes to lipid unsaturation by bypassing the cell's native lipid regulation pathway. We observed a surprising phenotype resulting from repression of the lipid desaturase-encoding OLE1: cellcell adhesion, termed flocculation, which we find is a transcriptional response to low membrane fluidity. Flocculation is induced when oxygen availability for lipid desaturation reactions is restricted and is part of a wide-ranging transcriptional response to low membrane fluidity that is activated during microaerobic fermentation.

\section{Materials and methods}

\subsection{Yeast strains}

All strains (Table1) are available from The Joint BioEnergy Institute (JBEI) public Inventory of Composable Elements (ICE): https://public-registry.jbei.org/.

Detailed plasmid and strain construction methods are available in the Supplemental Information.

\subsection{Growth and flocculation tests}

Aerobic growth of $O L E 1$-repressible strains was carried out in 24 well plates (Falcon) with $1 \mathrm{~mL}$ media at $30{ }^{\circ} \mathrm{C}$ using a microplate reader (BioTek Synergy 4; BioTek Instruments) with integrated temperature control and under continuous orbital shaking (slow shaking mode). The plates were sealed with a gas permeable adhesive seal (Thermo Fisher Scientific) to avoid evaporative losses of culture medium. These conditions were chosen to maintain consistent oxygenation of the cultures. Absorbance readings at $600 \mathrm{~nm}$ were used to characterize cell growth and detect flocculation via fluctuations in readings. Flocculation was also detected after $24 \mathrm{~h}$ of growth via 24 well plate imaging (UVP BioSpectrum Multispectral Imaging System) and confocal microscopy of cells in growth media stained with Calcofluor White at $23^{\circ} \mathrm{C}$. Micrographs were acquired on a LSM 710 (Zeiss) scanning confocal microscope with an oil immersion lens (100x magnification, 1.4 numerical aperture) using ZEN 2009 software (Zeiss).

Oxygen-limited fermentation growth of cells was carried out in a home-built apparatus based on $250 \mathrm{~mL}$ culture flasks fitted with rubber stopper. Caps were connected with tubing to a water bath, which allowed gas exflow via bubbling but restricted gas inflow. Flasks also featured syringe-fitted tubing for culture sampling at time points 
Table 1

Yeast strains used in this study.

\begin{tabular}{|c|c|c|}
\hline $\begin{array}{l}\text { Strain } \\
\text { name }\end{array}$ & Genotype & Reference \\
\hline BY4742 & MATa; his $3 \Delta 1 ;$ leu $2 \Delta 0 ;$ lys $2 \Delta 0 ;$ ura $3 \Delta 0$ & $\begin{array}{l}\text { (Brachmann } \\
\text { et al., 1998) }\end{array}$ \\
\hline JBEI-7971 & BY4742; ole1:: $\mathrm{P}_{\text {MET3 }}-O L E 1$ & This study \\
\hline JBEI-8010 & BY4742; spt23::kanMX ( $\Delta$ spt23) & This study \\
\hline JBEI-15533 & BY4742; mga2::URA3 ( $\Delta m g a 2)$ & This study \\
\hline JBEI-7989 & BY4742; flo1::yECitrine-URA3 $(\Delta$ flo1) & This study \\
\hline JBEI-7994 & JBEI-7971; flo1::yECitrine-URA3 ( $\triangle$ flo1) & This study \\
\hline JBEI-7991 & JBEI-7971; flo8::yECitrine-URA3 ( $\Delta$ flos) & This study \\
\hline JBEI-7990 & JBEI-7971; spt23::kanMX ( $\Delta$ spt23) & This study \\
\hline JBEI-14343 & JBEI-7971; mga2::URA3 (Amga2) & This study \\
\hline JBEI-16826 & JBEI-8010; pCM188-SPT23p90 & This study \\
\hline JBEI-16324 & JBEI-7989; pCM188(LEU2)-SPT23p90 & This study \\
\hline JBEI-16325 & JBEI-15533; pCM188(LEU2)-MGA2p90 & This study \\
\hline JBEI-14342 & JBEI-7971; pCM188-OLE1 & This study \\
\hline JBEI-14392 & JBEI-7971; pGREG523-P ${ }_{S P T 23^{-}}{ }^{\mathrm{myc}} S P T 23$ & This study \\
\hline JBEI-16629 & JBEI-7990; pGREG523-P ${ }_{S P T 23^{-}}{ }^{\text {myc }} S P T 23$ & This study \\
\hline JBEI-16824 & JBEI-7990; pGREG523-P ${ }_{S P T 23^{-}}{ }^{\mathrm{myc}} S P T 23_{\mathrm{W} 1042 \mathrm{~L}}$ & This study \\
\hline JBEI-16326 & JBEI-8010; pGREG523(2 $\mu)-\mathrm{P}_{S P T 23^{-}}{ }^{\mathrm{myc}} S P T 23$ & This study \\
\hline JBEI-16328 & 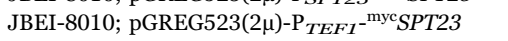 & This study \\
\hline JBEI-16327 & JBEI-7971; pGREG523-P ${ }_{M G A 2^{-}}{ }^{\text {myc }} M G A 2$ & This study \\
\hline
\end{tabular}

during growth. Cultures $(50 \mathrm{~mL})$ were briefly flushed with nitrogen before sealed after inoculation. Comparative aerobic growth was performed in $250 \mathrm{~mL}$ shake flasks with loose-fitting gas-permeable caps. Acute hypoxia was induced by thoroughly flushing aerobically growing cultures with argon and sealing their flasks with a tightly fitting rubber stopper connected to an argon-filled balloon to provide positive pressure.

\subsection{Fatty acid analysis}

Total lipids were routinely extracted from yeast cells harvested from $1 \mathrm{~mL}$ liquid culture using a modified Bligh Dyer method (Bligh and Dyer, 1959). Prior to lipid extraction, yeast cells were pelleted, resuspended in $400 \mu \mathrm{L} 0.9 \mathrm{M}$ Sorbitol/0.1 M EDTA (pH 7.0), and treated with 15 units zymolyase (Zymo Research) for $1 \mathrm{~h}$ at $35^{\circ} \mathrm{C}$. Lipids were extracted from the organic phase of a mixture of $1: 1: 1$ chloroform:methanol:water and dried under nitrogen. Lipid incorporated fatty acids were derivatized to their corresponding fatty acid methyl esters (FAMEs) by transesterification using $2 \%(\mathrm{v} / \mathrm{v})$ sulfuric acid in methanol $\left(90^{\circ} \mathrm{C} ; 2 \mathrm{~h}\right)$. FAMEs were subsequently extracted in $400 \mu \mathrm{L}$ hexane, of which $1 \mu \mathrm{L}$ was analyzed on an Agilent $5973-$ HP6890 GC-MS using a $30 \mathrm{~m}$ DB-5 ms capillary column. The oven was held at $40{ }^{\circ} \mathrm{C}$ for $3 \mathrm{~min}$, followed by a ramp to $300{ }^{\circ} \mathrm{C}$ at $20^{\circ} \mathrm{C} / \mathrm{min}$. The MS was operated in selected ion monitoring (SIM) mode using ions of $\mathrm{m} / \mathrm{z} 55$ and 74, which represent abundant and diagnostic fragment ions of saturated fatty acids (SFAs) and mono-unsaturated fatty acids (MUFAs), respectively. Abundance of fragment ions with $m / z 55$ was used for relative quantification of corresponding fatty acid species.

\subsection{Spectroscopy}

Fluorescence anisotropy analysis of membrane fluidity was carried out on $1 \mathrm{~mL}$ of cells in stationary phase that were pelleted, resuspended in $400 \mu \mathrm{L} \mathrm{0.9} \mathrm{M} \mathrm{Sorbitol/0.1} \mathrm{M} \mathrm{EDTA} \mathrm{(pH} \mathrm{7.0),} \mathrm{and} \mathrm{treated} \mathrm{with} 15$ units zymolyase (Zymo Research) for $1 \mathrm{~h}$ at $35^{\circ} \mathrm{C} .1 \%$ of a concentrated stock ( $5 \mathrm{mM}$ ) of di-phenyl-hexatriene (Sigma) in ethanol was added and the samples were incubated at room temperature for $30 \mathrm{~min}$. Steady-state anisotropy measurements were made on a Fluorolog spectrofluorometer (Horiba), equipped with automatic polarizers and a temperature controller. Fluorescent protein expression driven by the FLO1 promoter was monitored on a FACSAria II flow cytometer (BD Biosciences).

\subsection{Spt23p processing}

For Western blotting, total protein extracts were obtained from strains cultured at specified methionine concentrations using a urea/ SDS protein extraction method (Printen and Sprague, 1994). Protein extracts were separated by SDS-PAGE (7.5\% Mini-PROTEAN-TGX gels; BioRad), blotted onto a PVDF membrane and probed with 9E10 primary antibodies (mouse anti c-MYC; Developmental Studies Hybridoma Bank) and mAbGEa primary antibodies (mouse anti actin; Thermo Fisher Scientific) in PBST. Primary antibodies were detected with a goat anti-mouse IgG secondary antibody conjugated with horseradish peroxidase (Santa Cruz Biotechnology). Horseradish peroxidase activity was detected with SuperSignal ${ }^{\mathrm{TM}}$ West Pico Chemiluminescent Substrate (Thermo Fisher Scientific).

\subsection{Gene expression analysis}

To determine the relative expression levels of individual genes, RNA was extracted from cells in stationary phase cultured for flocculation assays using a commercial kit (YeaStar ${ }^{\mathrm{TM}}$; Zymo Research) according to manufacturer's instructions. RNA samples were treated with DNaseI (RapidOut DNA Removal Kit; ThermoFisher Scientific) to remove genomic DNA contaminations. One-step qRTPCR was carried out using a SYBR Green-based kit (Invitrogen) on a StepOnePlus Real-Time PCR System (Applied Biosystems). Primers were previously described by Van Mulders et al. (Van Mulders et al., 2009). Melting curve analysis was performed according to thermocycler specifications. Relative expression levels were analyzed with the $\Delta \Delta$ ct method and normalized to $A C T 1$ expression.

For global expression analysis, RNA-Seq libraries were prepared from cells at $\mathrm{OD}_{600} \sim 1$ grown in either SD (BY4742, aerobic or hypoxic), SD - uracil (BY4742 expressing plasmid-based p90 constructs), or SD - methionine ( $\mathrm{P}_{M E T 3^{-O L E 1}}$, with or without addition of $250 \mu \mathrm{M}$ methionine). In the case of $\mathrm{P}_{\text {MET3-OLE1 strains, growth }}$ was carried out in 24 well plates (as described above), with $24 \times 1 \mathrm{~mL}$ cultures pooled together per sample. BY4742 cells were grown in $250 \mathrm{~mL}$ flasks as described above. After growth, cells were briefly spun down (5000 rpm, $1 \mathrm{~min}$ ) and immediately frozen in liquid nitrogen. Total RNA was extracted from frozen pellets via the hot phenol method (Collart and Oliviero, 2001), and mRNA was isolated via poly-A beads (New England BioLabs). From these, Illumina RNA-Seq libraries were prepared with the adapter ligation method (NEBNext Ultra RNA Library Prep Kit for Illumina, New England BioLabs) and barcoded with index oligonucleotides (NEBNext Multiplex Oligos for Illumina, New England BioLabs). Samples were pooled, and sequencing was performed on either an Illumina HiSeq. 2000 ( $\mathrm{P}_{\text {MET3 }}$-OLE1 samples, $\sim 2 \mathrm{M}$ reads per sample) or MiSeq (p90s and hypoxia samples, $\sim 1 \mathrm{M}$ reads per sample). HiSeq runs were performed at the Vincent J. Coates Genomics Sequencing Laboratory at UC Berkeley, supported by NIH S10 Instrumentation Grants S10RR029668 and S10RR027303, while MiSeq runs were performed in-house at the Joint BioEnergy Institute. Sequencing reads were aligned to the S288C R64-1-1 reference genome with TopHat 2 (version 2.0.13) with a max intron length of 2500 and differential analysis was performed with Cuffdiff (version 2.2.1) with the -frag-bias-correct and the -multi-read-correct flags. Infinite values for differential expression resulting from assigned reads in one condition were assigned a $\log _{2}$ value of 10 . Statistical analysis and plotting was done in R. Gene Ontology (GO) term mapping was done with PANTHER (version 10.0; http://www.pantherdb.org) according to PANTHER Classification System in terms of "biological processes" in which respective gene products are involved. A processed data file containing all differential expression values and the raw sequencing data are publicly available at the Gene Expression Omnibus (https:// www.ncbi.nlm.nih.gov/geo) using the GEO accession number GSE94345. 


\section{Results}

\subsection{Construction and characterization of a titratable unsaturated lipid strain}

We screened different constitutive and repressible promoters (Fig. $\mathrm{S} 1)$ to effectively replace the native $O L E 1$ promoter, aiming to generate a system that can sample a wide range of phospholipid unsaturation stoichiometry (\% of acyl chains with double bonds). One of these was the MET3 promoter ( $\mathrm{P}_{\text {MET3 }}$ ), which has previously been characterized as being tightly controlled by methionine concentrations in the media (Mao et al., 2002) and has been used to regulate expression of an essential ergosterol synthesis gene in yeast (Asadollahi et al., 2008; Paradise et al., 2008). Substitution of the endogenous OLE1 promoter with the MET3 promoter yielded an unsaturated fatty acid auxotroph on agar plates supplemented with methionine (Fig. 1B). In liquid media, lipid composition of growing cells was dependent on the concentration of methionine in the media, with the proportion of lipid acyl chains with double bonds ranging from $25 \%$ to greater than $80 \%$ (Fig. 1D), as measured by gas chromatography - mass spectrometry (GC-MS) of derivatized fatty acids extracted from cell lipids (Fig. 1C). To test the effects of lipid unsaturation on membrane fluidity, we used the fluorescence anisotropy of di-phenyl-hexatriene (DPH). The emission anisotropy of this probe reflects its thermal motion in the host membrane (Lentz, 1989). Anisotropy measurements on yeast $\mathrm{P}_{\text {MET3- }}{ }^{-}$ $O L E 1$ spheroplasts incubated with DPH yielded an increasing ordering parameter (R), reflecting lower membrane fluidity, when grown at increasing methionine concentrations (Fig. 1E). This effect could be partially relieved by the addition of the established membrane fluidizer n-octanol (Heipieper et al., 2000) to the spheroplasts (Fig. 1E). This strain therefore serves as a cellular model to monitor effects of decreasing membrane fluidity when grown under varying concentrations of methionine.

\subsection{Membrane fluidity regulates yeast flocculation through Spt23p mediated activation of FLO1}

While characterizing the effect of lipid unsaturation on exponential growth rates (Fig. S2A,B,C), we observed an unexpected phenotype: cells with low lipid unsaturation visibly clumped together, forming macroscopic aggregates that precipitated from the medium (Fig. 2A). Micrographs (Fig. S2D) showed that clumping was not due to any sort of defects in cell division (e.g. chain formation (Stratford, 1992a) or agglomeration (Guinard and Lewis, 1993), but rather resulted from binding of neighboring yeast cells through cell wall interactions. We were able to restore non-adhesive growth by the addition of exogenous oleic acid to the culture during growth (Fig. S3A), as well as by plasmid-based expression of OLE1 (Figs. 2B; S4A), demonstrating that the flocculation is induced by modified lipid composition. Cell aggregation was blocked when D-mannose was added to the culture medium post-growth, consistent with a role for a class of mannose-binding proteins, termed flocculins, that cause adhesion between the polysaccharide-coated cell walls of neighboring yeast cells (Stratford and Assinder, 1991). Other monosaccharidic hexoses as well as disaccharidic maltose had no effect on flocculation (Fig. 2D). We also observed reduced flocculation when cells were grown in the presence of established membrane fluidizers $n$-octanol (oct; $0.02 \% \mathrm{w} / \mathrm{v}$ ) and benzyl alcohol (Mukhopadhyay et al., 2002; Sinicrope et al., 1992) (BnOH; $10 \mathrm{mM}$ ) (Fig. 2C, Fig. S3B), though these did not affect lipid unsaturation (Fig. S4B). This indicated that low membrane fluidity, and not lipid composition per se, were responsible for flocculation. Flocculation was unaffected when these additives were introduced post-growth (Fig. 2C), as was the case for oleic acid (Fig. 2D), suggesting that flocculation resulted from a transcriptional response to low membrane fluidity.

We screened expression changes upon OLE1 repression for a set of flocculation $(F L O)$ genes and found a significant increase in expression of a single flocculation gene, FLO1 (Fig. S3C). Flo1p is a GPI-anchored cell wall mannose binding protein, and thus is functionally consistent with the dispersion of flocs in high concentrations of D-mannose (Fig. 2D). However, the activation of FLO1 expression was surprising in the S288C strain background, which features an in-frame stop codon in FLO8, encoding the major transcription factor activating flocculation. This allele (flo8-1, G425A) has been proposed to have been selected for during strain cultivation due to the difficulty of working with flocculent strains in the lab (Liu et al., 1996). We confirmed that lipid-triggered flocculation was independent of the canonical pathway by knockout of FLO8, which did not affect flocculation (Fig. 2E) nor lipid composition (Fig. S4C). In contrast, deletion of FLO1 completely abolished flocculation (Fig. 2E) and did not affect lipid unsaturation (Fig. S3C). In this deletion strain, FLO1 was replaced with a fluorescent reporter gene (yECitrine), which allowed us to monitor the increase in $\mathrm{P}_{\text {FLO1 }}$ activity as lipid unsaturation dropped from $80 \%$ to $45 \%$ (Fig. 2F).

The observations that membrane-fluidizing additives inhibit flocculation led us to hypothesize that activated forms (p90 domains) of known membrane fluidity sensors (Spt23p and Mga2p) directly initiate FLO1 transcription, as is the case for OLE1. Previous studies could only observe reductions in Spt23p/Mga2p proteolytic processing by the supplementation of the growth media with highly-fluidizing polyunsaturated fatty acids, which are not found in S. cerevisiae (Hoppe et al., 2000; Jiang et al., 2002). We asked if genetic titration of OLE1 would instead modulate Spt23p/Mga2p processing in response to varying levels of native unsaturated lipids. We first introduced a $\mathrm{N}$-terminally 13xmyc-tagged Spt23p variant $\left({ }^{\mathrm{myc}} \mathrm{Spt} 23 \mathrm{p}\right)$, whose proteolysis can be monitored by Western blotting (Fig. 3A), on a centromeric plasmid. Spt23p processing and release of p90 domains was induced when OLE1 was repressed in the presence of methionine, as indicated by a relative enrichment of ${ }^{\mathrm{myc}} \mathrm{Spt} 23 \mathrm{p} 90$ over ${ }^{\mathrm{myc}} \mathrm{Spt} 23 \mathrm{p}$ (Fig. 3B). Importantly, ${ }^{\mathrm{myc}} \mathrm{SPT} 23$ was expressed using its endogenous promoter $\left(\mathrm{P}_{\text {SPT23 }}\right)$, as overexpression of ${ }^{\mathrm{myc}} \mathrm{Spt} 23 \mathrm{p}$ using a strong promoter or a high copy plasmid led to constitutive FLO1 induction (Fig. S5B). Similar processing was observed in an $\Delta s p t 23$ background (Fig. S5A) and for ${ }^{\mathrm{myc}} \mathrm{Mga} 2 \mathrm{p}$ (Fig. S5C). In the $\Delta$ spt23 background, proteolytic processing could be completely abolished using a W1042L variant of ${ }^{\mathrm{myc}}$ Spt23p (Fig. 3B), supporting the importance of this residue in a rotation-based activation mechanism of Spt23p (Covino et al., 2016). These data therefore provide further evidence that Spt23p and Mga2p act as membrane fluidity sensors that respond to changes in native lipid composition (Hoppe et al., 2000; Nakagawa et al., 2002).

We next assayed genetic roles for SPT23 and its paralogue, MGA2, in OLE1-mediated FLO1 expression and flocculation. Because OLE1 is freed from its endogenous promoter in the $\mathrm{P}_{M E T 3}$-OLE1 background, we were able to delete SPT23 while maintaining the engineered strain's control of lipid unsaturation with supplemented methionine (Fig. S4D). Deletion of SPT23 strongly reduced flocculation (Fig. 3C) and FLO1 expression (Fig. 3D); flocculation could be fully restored by plasmidbased expression of a SPT23 variant $\left({ }^{\mathrm{myc}} \mathrm{Spt} 23 \mathrm{p}\right.$, Fig. S5A). MGA2 deletion prevented flocculation as well, but also strongly inhibited cell growth in the presence of external methionine (Fig. 3C, D), and thus its effects on flocculation could not be fully characterized. We reasoned this null phenotype could be a result of a potential additional, posttranscriptional role for Mga2p in stabilizing OLE1 transcripts (Kandasamy et al., 2004), which would act independently of our OLE1 promoter replacement strategy. To compare the effects of Spt23p and Mga2p on FLO1 expression, we instead expressed truncated gene products containing only active p90 domains, simulating the proteolysis that activates them endogenously (Auld et al., 2006). When these domains were expressed from a repressible promoter (tet $\mathrm{O}_{2}-\mathrm{P}_{C Y C 1}$ ), we observed flocculation in wild-type cells that was abolished by addition of the repressor (doxycycline). Flocculation was induced more strongly by Spt23p90 in comparison to Mga2p90, and 
A

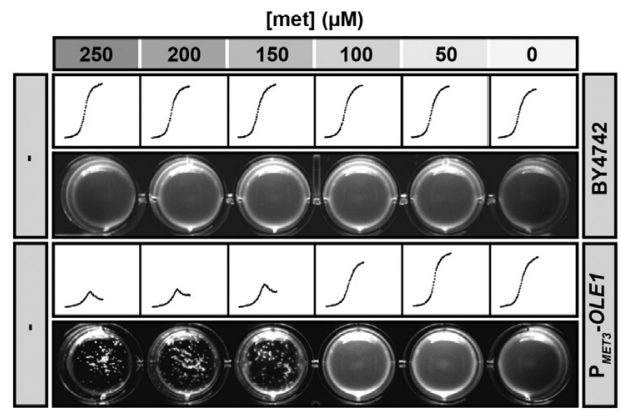

B

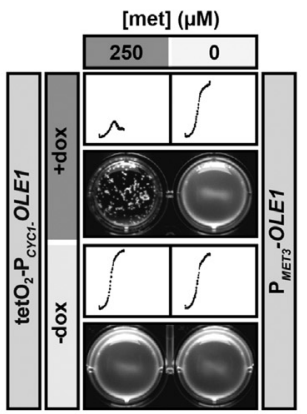

C

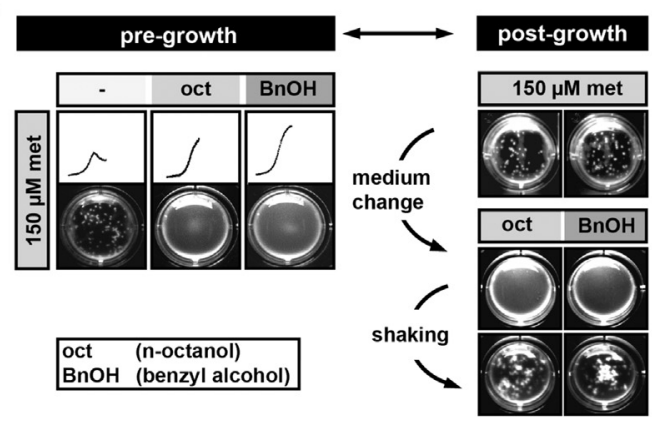

D

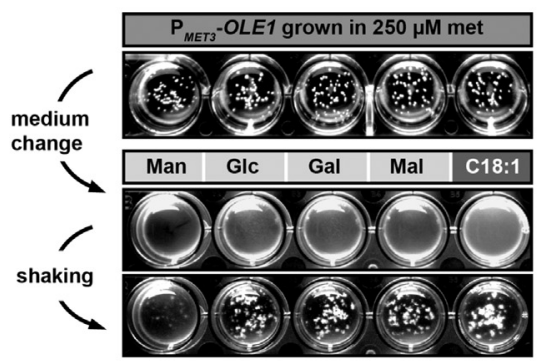

E

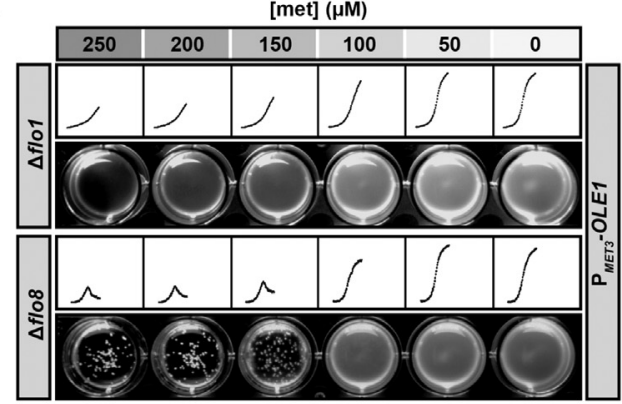

F

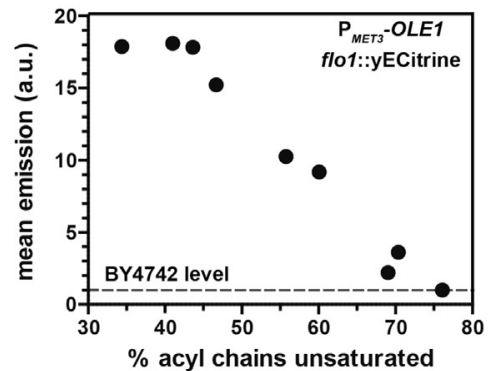

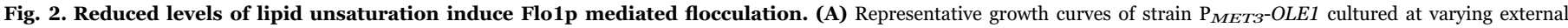

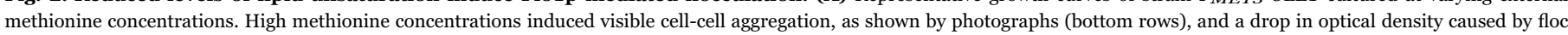

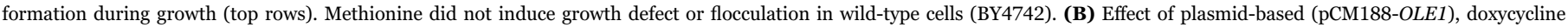

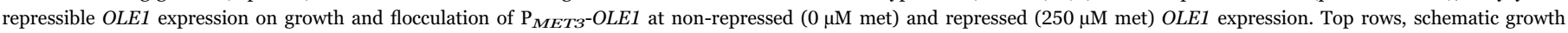

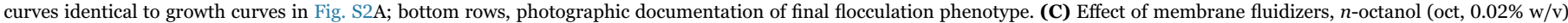

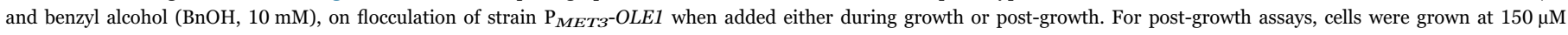

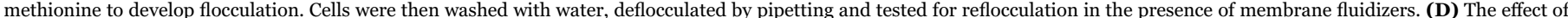

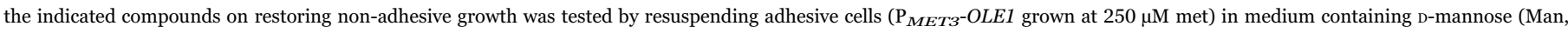

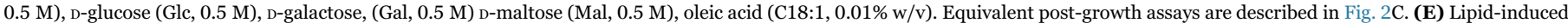

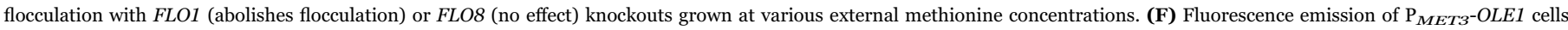

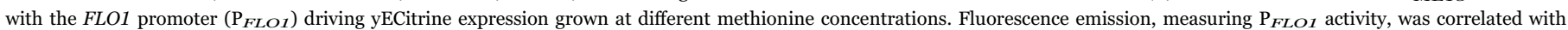
unsaturated lipid stoichiometry.

was abolished completely by deletion of FLO1 (Fig. 3E). These flocculation strengths corresponded to differences in FLO1 expression upon induction of Spt23p90 and Mga2p90 (Fig. 3F). Spt23p and Mga2p therefore act upon FLO1 expression during low lipid unsaturation, with Spt23p serving as the dominant activator.

\subsection{Lipid mediated flocculation is triggered by oxygen limitation during fermentation}

We next sought to identify potential physiological roles for reduced lipid desaturation activating FLO1. For S. cerevisiae strains used in brewing, flocculation allows for industrial-scale clarification of the culture (wort) without the use of filtration or centrifugation. Fermentation vessels, unlike laboratory flasks, feature restricted architecture meant to reduce oxygen availability in the culture. While yeast grown anaerobically are strict unsaturated fatty acid auxotrophs (Andreasen and Stier, 1954), we hypothesized that such a microaerobic environment could restrict Ole1p activity through reduced oxygen binding and availability for the desaturase reaction. To test whether oxygen limitation could drive flocculation, we grew wild-type cells (BY4742) in minifermentors that allow for outflow of gas (e.g. carbon dioxide), but not oxygen uptake (Fig. 4A). Growth of cells under these conditions resulted in the increased accumulation of ethanol, i.e. fermentation, compared to well-aerated growth in standard shake flasks (Fig. $4 \mathrm{~B}$ and $\mathrm{C}$ ) at the expense of biomass $\left(\mathrm{OD}_{600}\right.$ of $2.3 \pm 0.06$ vs. $2.9 \pm 0.06$ after $48 \mathrm{~h}$ ). As fermentation progressed, we observed triggering of visible flocculation similar to that observed during $O L E 1$ repression. This did not occur when the culture was well aerated in a standard shake flask, nor in the absence of FLO1 or SPT23 (Fig. 4A).
The increase in FLO1 expression under hypoxic growth (Fig. 4E) mirrored the reduction in lipid unsaturation as fermentation progressed (Fig. 4D), as monitored by the shift from glucose to ethanol in the culture. Under these conditions, unsaturated lipid stoichiometry was reduced to $45 \%$ of all acyl chains, corresponding to the levels where we observe full $\mathrm{P}_{F L O 1}$ induction and Spt23p processing in

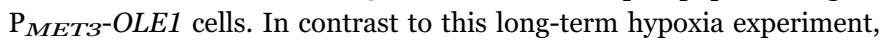
we found that growth of cells under short-term, strict oxygen starvation, achieved by incubation under argon for $90 \mathrm{~min}$, did not lead to increased FLO1 expression (Fig. S6A). Because Ole1p acts during de novo lipid synthesis, we reasoned that only long-term exposure to hypoxic conditions would lead to significant changes in cell lipid composition (Fig. S6B), and therefore serves as a trigger for flocculation.

\subsection{Membrane fluidity-regulated genes are globally activated during microaerobic fermentation}

The central and surprising role of lipid composition in regulating a non-membrane process (cell wall adhesion) motivated us to ask if this was indicative of a larger long-term hypoxic response regulated by membrane fluidity. We first used deep sequencing of mRNA-derived cDNA (RNA-Seq) to characterize changes in global gene expression upon OLE1 repression. Cells $\left(\mathrm{P}_{\text {MET3 }}-O L E 1\right)$ grown in the presence of $250 \mu \mathrm{M}$ methionine $(38.4 \pm 2.5 \%$ unsaturated lipids $)$ were sampled after $24 \mathrm{~h}$ of growth and were compared to those with no added methionine ( $88.3 \pm 2.3 \%$ unsaturated lipids). OLE1 repression led to large-scale gene expression changes, with 1012 genes significantly (qvalue $\leq 0.05$ ) upregulated and 882 downregulated (Fig. 5A). Systematic 
A

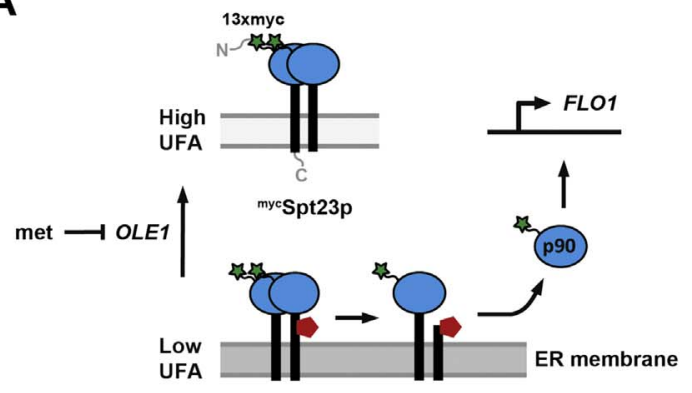

B

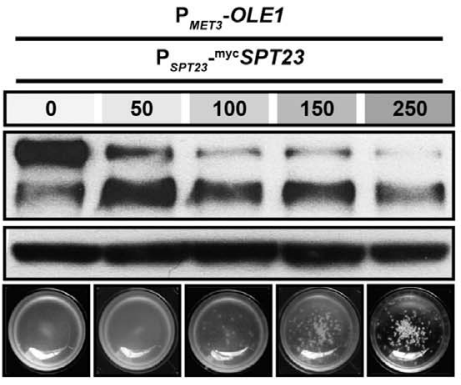

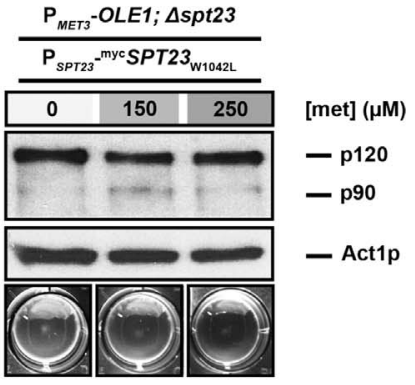

C

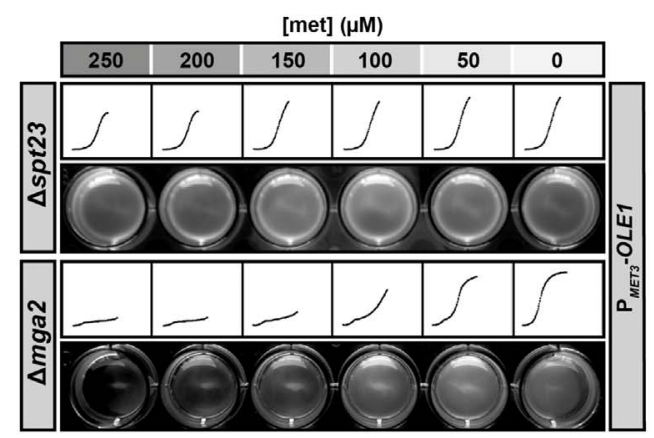

D

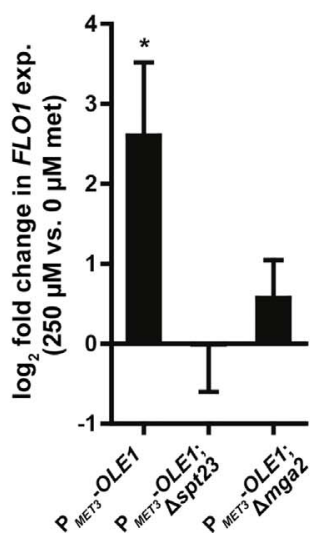

E

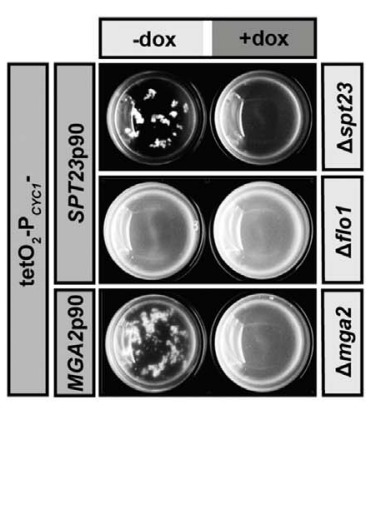

$\mathbf{F}$

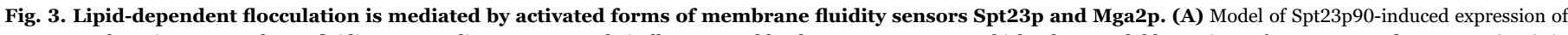

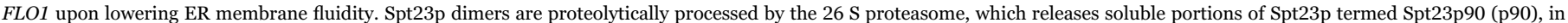

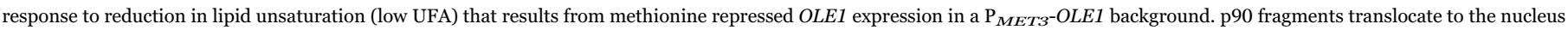

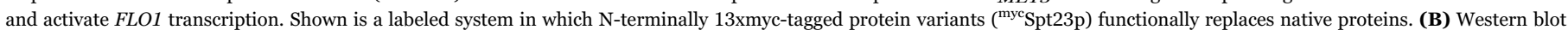

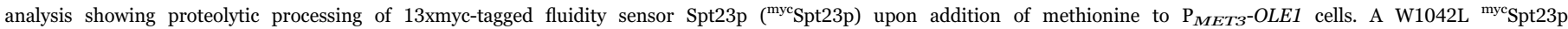

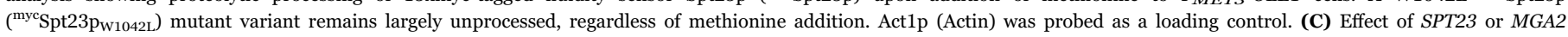

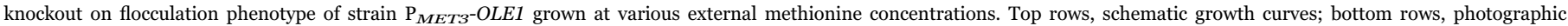

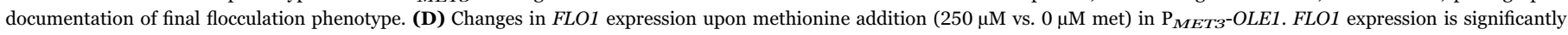

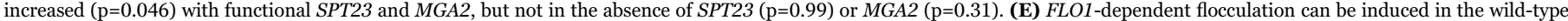

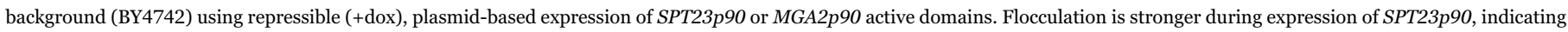

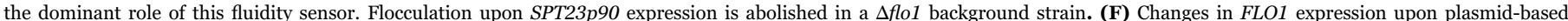

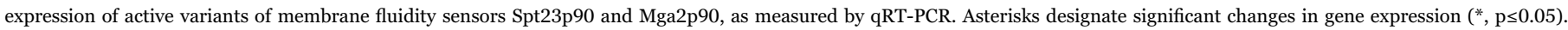
Error bars, SEM ( $\mathrm{n}=3$ ).

GO term mapping of upregulated genes upon OLE1 repression showed significant enrichment of those involved in lipid and fatty acid metabolism, glycolysis and carbohydrate metabolism, and response to stress stimuli (Fig. S7D). FLO1 was one of the strongest overexpressed genes upon OLE1 repression, with a $\log _{2}$ differential expression value ( $\left.\log _{2} \mathrm{DE}\right)$ of 5.0. In contrast, downregulated genes include those involved in protein and ribosome synthesis (Fig. S7D), consistent with non-specific effects of OLE1 repression in slowing growth. We reasoned that transcriptional changes triggered by OLE1 repression were largely a result of physiological responses to changes in membrane homeostasis, as opposed to specific activation by lipidactivated transcriptional elements, and could also contain artifacts from reduced growth rates and methionine supplementation. We therefore also characterized expression effects to the induction of active Spt23p90 and Mga2p90 domains. Of the 1012 OLE1-sensitive genes, 341 were also significantly activated by either Spt23p90 or Mga2p90 (Fig. 5B). For FLO1, expression was increased by both activators, though more strongly by Spt23p90 $\left(\log _{2}\right.$ DE of 3.7 vs. 2.4). Among all genes significantly overexpressed by either $\mathrm{p} 90$ domains, there was only a weak correlation between the increase in expression in response to the individual domains (e.g. Mga2p90 vs. Spt23p90, Pearson $r=0.19$ ). Along with the paucity of genes activated by both Mga2p90 and Spt23p90 domains ( 15\% of the genes activated by either), this indicates a divergent set of targets for these paralogues, despite their presumably similar modes of activation.

Changes in lipid unsaturation have a role in activation of FLO1 during oxygen limitation; to what extent does this pathway play a role in activation of other Spt23p/Mga2p targets during extended hypoxic growth? To generate a comparative data set, we analyzed global expression profiles for wild-type cells (BY4742) grown aerobically compared to those grown under long-term hypoxic conditions (e.g., $18 \mathrm{~h}$, conditions described above) or under acute hypoxia (90 min under argon); these conditions mimicked those for FLO1 expression (Fig. 4). Long-term hypoxia caused widespread changes in gene expression: 1373 upregulated genes with Gene Ontology (GO) terms significantly enriched in the metabolism of coenzymes, vitamins, and lipids (Fig. S7D), including OLE1 $\left(\log _{2} \mathrm{DE}\right.$ of 3.2). In contrast, acute hypoxia induced a significantly smaller transcriptional response (266 upregulated genes), yet still included several canonical hypoxic response elements (e.g. TIR1 and ANB1). These profiles are consistent with previous characterizations of gene-dependent timescales for hypoxia response induction upon oxygen starvation responses in $S$. cerevisiae, which was interpreted as suggesting the activity of multiple oxygen sensors (Kwast et al., 1998). Of the set of genes identified as being sensitive to $O L E 1$ repression and induced by Spt23p90 or Mga2p90 domains, 70\% were significantly upregulated during longterm hypoxic growth (Fig. 5C). Differential expression values for longterm hypoxic growth were significantly correlated with differential 
A

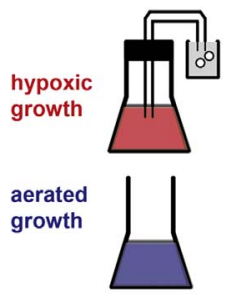

B

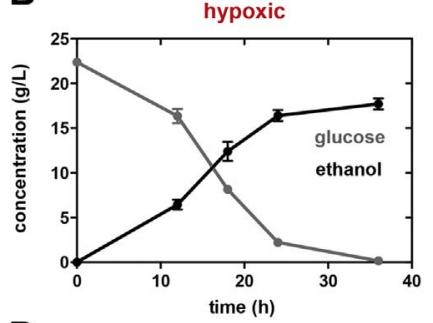

D

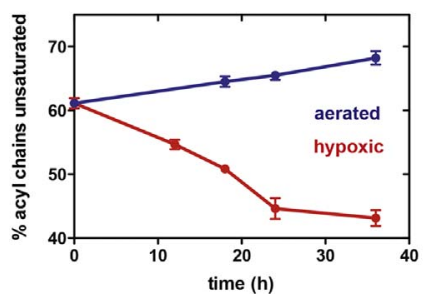

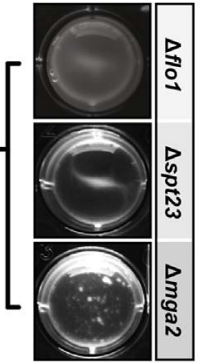

C
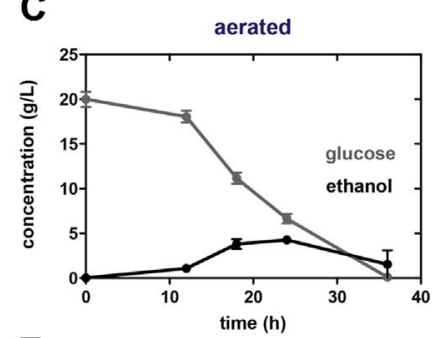

E

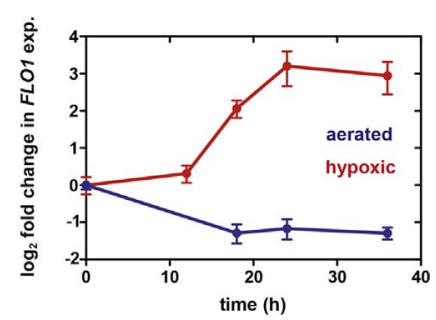

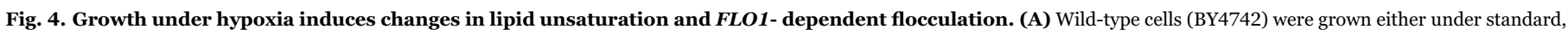

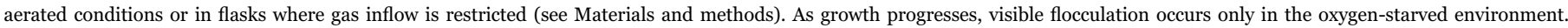

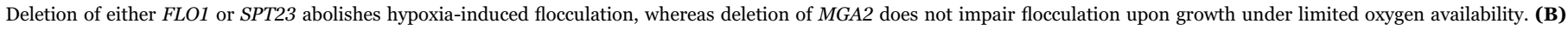

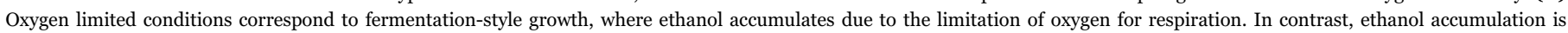

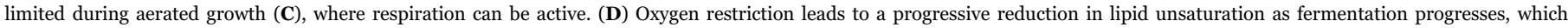
corresponds to an increase in FLO1 expression (E), explaining the flocculation phenotype in this environment. Error bars, SEM (n =3).

expression upon OLE1 repression (Fig. S7A, r=0.38, $\mathrm{p}=2 \times 10^{-13}$ ) and for the additive effects of Spt23p90 and Mga2p90 induction (Fig. 5D, $\mathrm{r}=0.49, \mathrm{p}<10^{-15}$ ), which is consistent with a model in which the two activators have independent effects on transcription (Herschlag and Johnson 1993). These correlations are medium in strength, which likely reflects additional roles for other hypoxic response pathways. In contrast, differential expression values for short-term (90 min) oxygen starvation were not significantly correlated with either differential expression upon OLE1 repression ( $\mathrm{p}=0.07$ ) (Fig. S7B) or upon $\mathrm{p} 90$ induction ( $\mathrm{p}=0.5$ ) (Fig. $\mathrm{S} 7 \mathrm{C}$ ). The ER membrane fluidity regulon is therefore induced by long-term hypoxia in yeast and plays an extensive role in the transcriptional response to fermentation conditions.

\section{Discussion}

The experiments presented here use rational genetic manipulation of lipid desaturase expression in order to allow for causal study of the roles for lipid unsaturation and membrane fluidity in the physiology of budding yeast. Modulation of $O L E 1$ promoter activity allowed us to characterize the dependence of yeast physiology on lipid unsaturation. Most strikingly, we identified a role for lipid unsaturation in regulating yeast cell-cell adhesion or flocculation. Flocculation is a well-known aggregation phenotype among different yeast species with a large set of diverse stress conditions, including nutrient limitation and starvation (Straver et al., 1993; Soares et al., 1994; Smit et al., 1992), temperature and pH stress (Soares et al., 1994; Stratford, 1992b), and cell aging (Soares and Mota, 1996; Powell et al., 2003; Barker and Smart, 1996). Because native flocculation pathways have generally been studied in yeast species and strains with heterogeneous genetic backgrounds, there has been an assumption that flocculation behavior is a highly strain-specific phenomenon (Verstrepen et al., 2003). Due to a nonsense mutation in the FLO8 gene (Liu et al., 1996), previous studies showing flocculation in the S288C background relied on restoring Flo8p activity (Liu et al., 1996; Bester et al., 2006), direct expression of different flocculins (Van Mulders et al., 2009; Govender et al., 2008), or overexpression of related factors Gts1p (Shen et al., 2006) or Mss11p (Bester et al., 2006). Our work presents a novel, FLO8-independent pathway for inducing flocculation, in which reduction in unsaturated lipid content causes the activation of ER-bound membrane fluidity sensors, primarily Spt23p, that induce FLO1 expression. This pathway is triggered by genetic repression of $O L E 1$, but also by hypoxia (Fig. 4), as desaturase activity requires molecular oxygen. It is likely that other stimuli that are expected to reduce membrane fluidity or lipid desaturase activity, e.g. low temperature (Nakagawa et al., 2002), iron depletion (Vasconcelles et al., 2001), and transition metals (Kwast et al., 1999), can also induce flocculation by increasing Spt23p processing.

We propose that the regulation of FLO1 by lipid composition can account for many of the aggregation features utilized for separating cells from the fermentation broth (or wort) in industrial fermentation. For example, in brewing, initial culture aeration is a long-recognized factor that modulates the extent and timing of flocculation (Straver et al., 1993) and studies with both beer and wine producing yeast have reported that supplementation of the growth medium with ergosterol and fatty acids counteracts the consequences of anaerobic conditions (Straver et al., 1993; Holmberg, 1978; Tesniere et al., 2013). In this study we present a molecular mechanism that connects oxygen availability and flocculation mediated by the physical state of the ER membrane. In poorly aerated fermenters, oxygen levels are reduced as the culture grows, thereby reducing lipid desaturation reactions and 
A
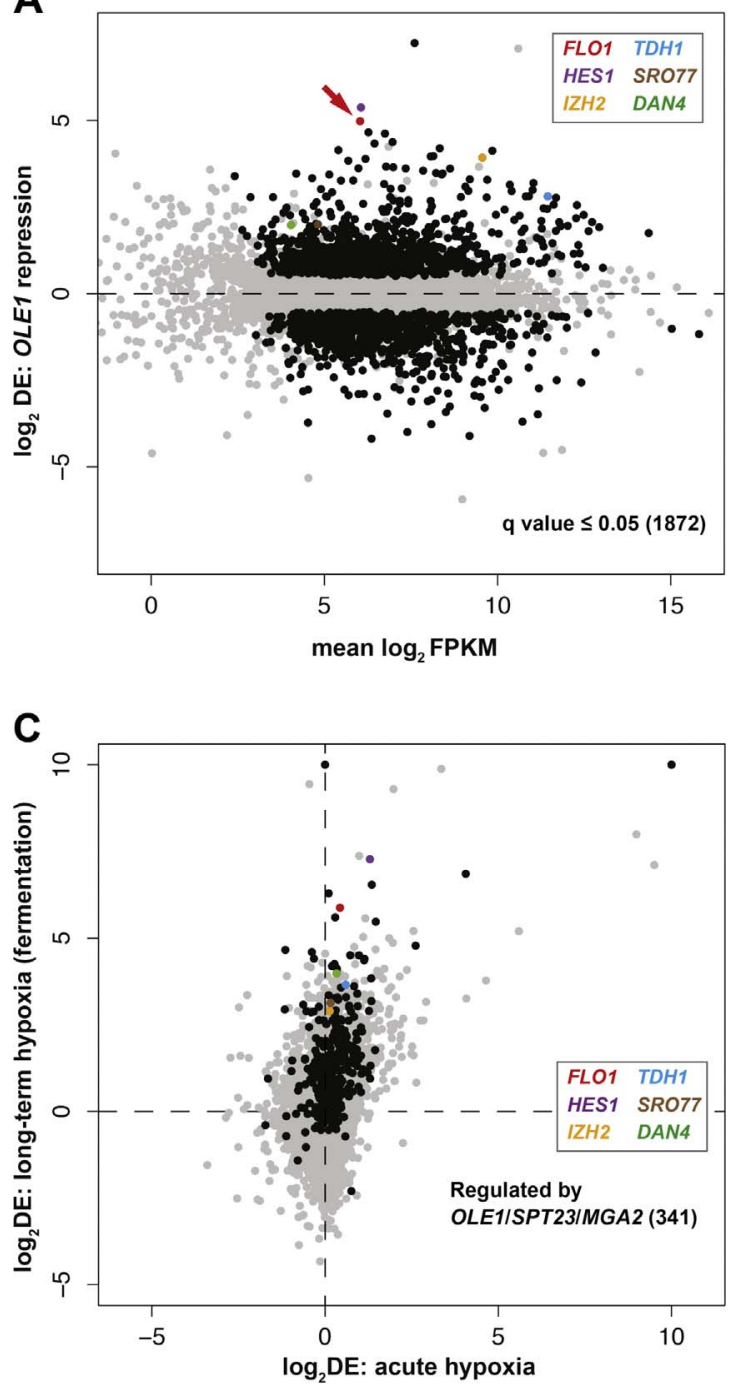

B
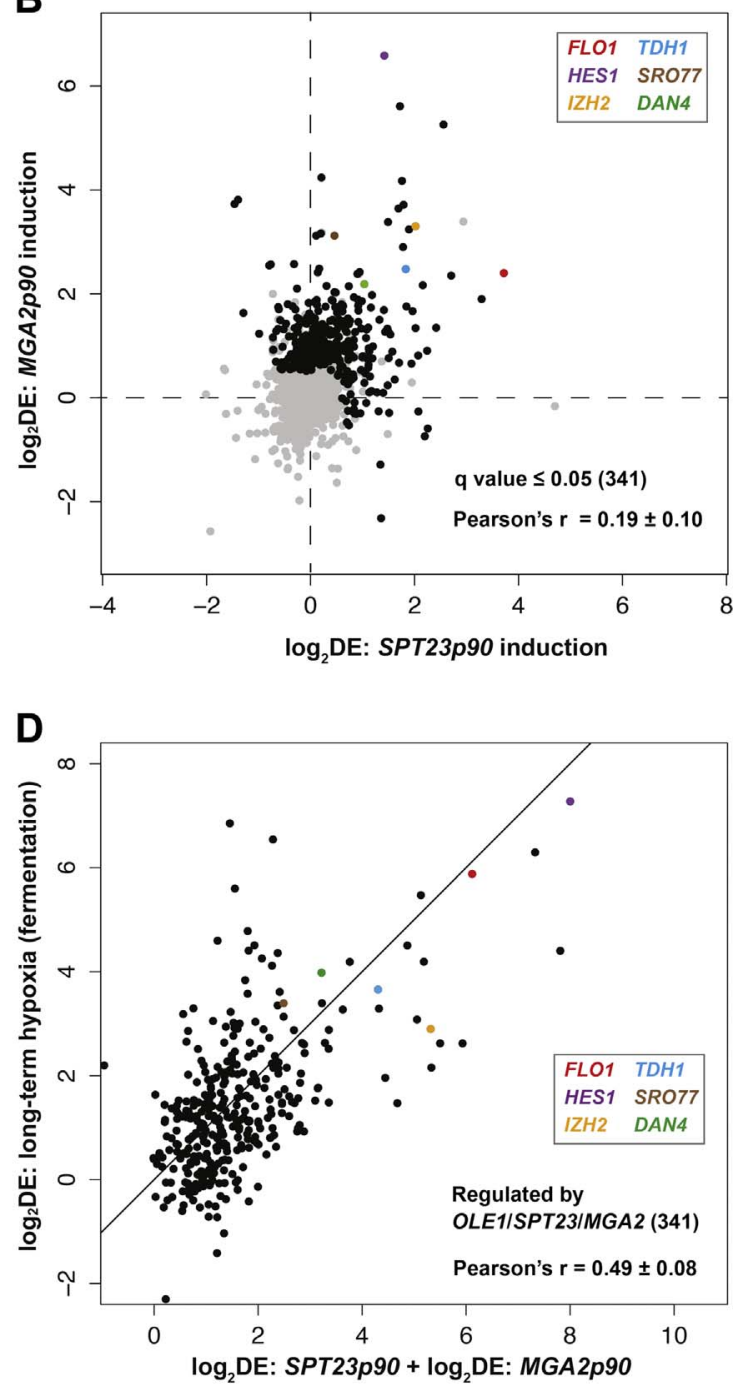

$\mathbf{E}$

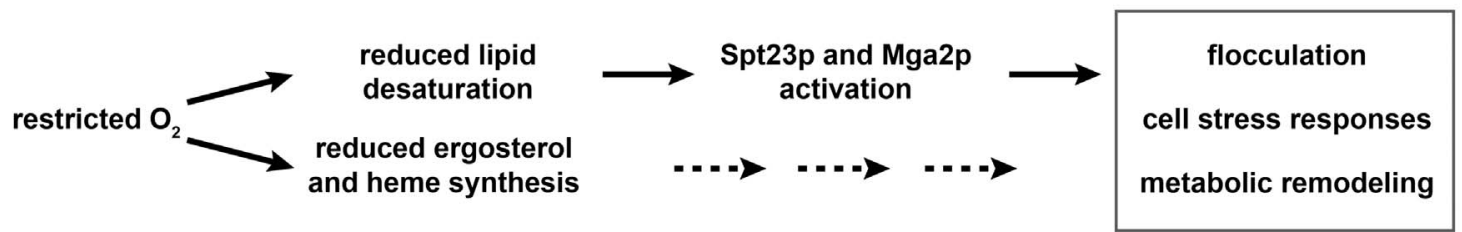

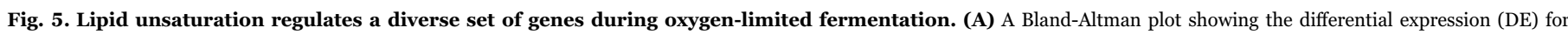

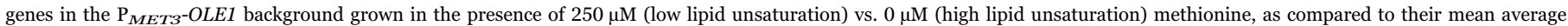

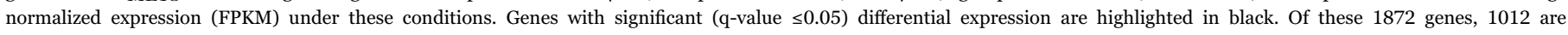

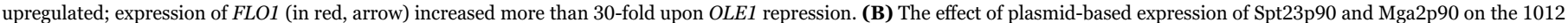

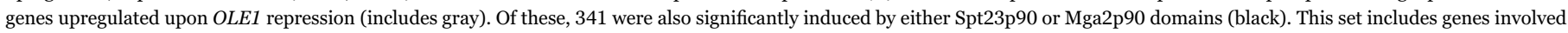

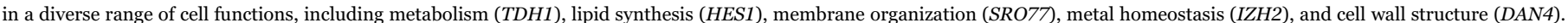

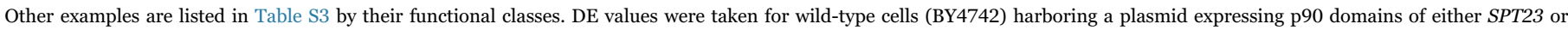

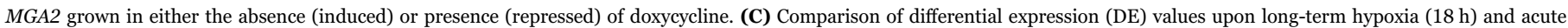

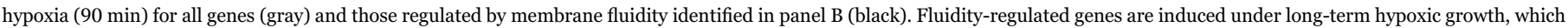

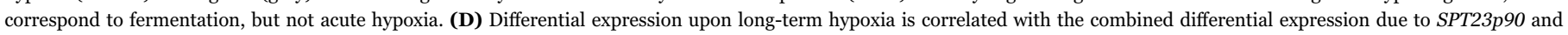

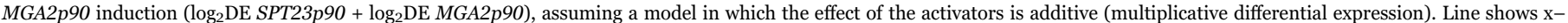

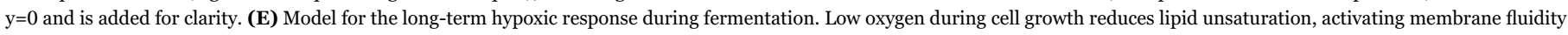

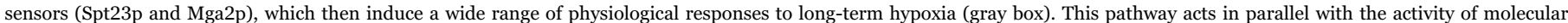
sensors for sterols and heme, whose synthesis is also oxygen dependent.

increasing $\mathrm{FLO1}$ expression. In this context, the late stage switch from glycolytic to respiratory metabolism would especially starve cells and their lipid desaturases from oxygen, thereby providing the trigger for flocculation to increase at the end of fermentation. Our work therefore provides a mechanistic understanding of the activation of flocculation due to oxygen restriction and its timing during fermentation. This is an important parameter for industrial yeast, as premature flocculation causes incomplete or 'hanging' fermentation and, in the context of brewing, severe off-flavors (Stratford, 1992b; Verstrepen et al., 2003). Future work will utilize the genetic components and lipid-mediated 
pathway characterized here to introduce and tailor flocculation behavior in industrial strains.

What physiological benefits for cell aggregation under unsaturated lipid restriction, potentially initiated by oxygen limitation, could lead the evolution of this flocculation pathway? Experiments on laboratory yeast overexpressing FLO1 show that flocculating cells are physically shielded from harmful effects of the external milieu by diffusion barriers built up within flocs (Smukalla et al., 2008). Flocculation, which is routinely triggered by nutritional starvation in FLO8 cells, is widely thought of as an adaptive stress response. In the case of oxygen starvation, however, floc formation would appear to be disadvantageous, as aggregates minimize surface area for diffusive uptake of oxygen. On the other hand, such diffusion barriers could generate microenvironments with high local concentrations of limiting nutrients, such as unsaturated fatty acids and sterols, which are released by lysing cells inside of flocs (Iserentant, 1996; Steward and Russell, 1981). Alternatively, flocculation could present a strategy for increasing the efficiency of mating, which is itself induced by stress conditions e.g. nitrogen limitation. In this model, flocculation is a prelude to mating by increasing the proximity of neighboring haploid yeast cells (Goossens et al., 2015). Our observations that Spt23p90 and Mga2p90 also activate transcription of a number of meiotic cell cycle and sporulation genes (Table S3) support such an interplay between unsaturated lipid composition and mating in yeast, which is potentially related to their originally proposed role in mating silencing (Dula and Holmes, 2000). In light of the possible commercial origins of laboratory yeast strains (Mortimer and Johnston, 1986), we also consider the role of saturated lipid and hypoxia-induced flocculation during industrial fermentation. In brewing, ale strains ( $S$. cerevisiae) are known as top-fermenters, as flocs rise to the surface of wort due to association with carbon dioxide bubbles arising from alcohol fermentation. In this context, flocculation could assist in oxygen uptake by bringing the yeast closer to the aerated surface. This would suggest the pathway presented here is a highly strain specific phenomenon. However, we noted a mention that OLE1 repression also results in cell aggregation in the distantly related, pathogenic fungus Candida albicans (Krishnamurthy et al., 2004). Lipid-regulated flocculation pathway could therefore be conserved among non-brewing yeast species, which are similarly equipped with all required components involved in the signal pathway (Oh and Martin, 2006).

The central role of Spt23p in regulating FLO1 expression is in line with a model in which membrane fluidity sensors act not only to maintain unsaturated lipid homeostasis but also as general regulators of cell physiology in response to environmental factors. Several lines of previous evidence support this proposal, including the discovery of SPT23 and MGA2 as silencers of mating genes (Dula and Holmes, 2000), the finding that Mga2p activates a minimal Low Oxygen Response Element (LORE) promoter (Jiang et al., 2001), and the detection of potentially hundreds of physical interaction sites for active Spt23p/Mga2p fragments using chromatin immunoprecipation DNA microarray (ChIP-chip) (Auld et al., 2006). We took advantage of the strains made to analyze $O L E 1$-dependent flocculation to carry out a genome-wide characterization of genes regulated by membrane fluidity. This effort yielded a large set of genes ( $15 \%$ of genome) induced by low lipid unsaturation, as well as a subset that are also activated by heterologous expression of pre-processed Spt23p/Mga2p active domains (341 genes). Manual curation of our expression data reveals several functional categories for genes activated by low membrane fluidity, which are summarized in Table S3. Common targets include synthesis and regulatory genes (involved in the synthesis of fatty acids, phospholipids, sterols, sphingolipids, and lipid droplets) and genes involved in metal homeostasis and tolerance, consistent with the catalytic roles for iron and the inhibitory roles of other metals on lipid metabolism (Pagani et al., 2007; Lyons et al., 2004). Repression of OLE1 or expression of Spt23/Mga2 p90 also induced a number of stress response genes involved in osmotic shock, cold shock, heat stress, and oxidative stress, as well as members of well-characterized cell protein homeostasis pathways: the Heat Shock Response, the Unfolded Protein Response, and ER Associated Degradation. Finally, we note a prominence of fluidity-regulated genes that encode for catalytic and structural proteins involved in mitochondrial structure and respiratory metabolism, which motivate future experiments on the role of unsaturated lipids in the dynamics and inheritance of these organelles (Stewart and Yaffe, 1991).

Our global expression analysis supports the hypothesis that the level of unsaturated lipids serves as broad regulator of gene expression during oxygen-limited fermentation. The membrane fluidity regulon is globally induced upon hypoxic growth (Fig. 5C,D and Fig. S7A), and includes classic hypoxia genes (DAN4, TIR genes), those associated with the diauxic shift (ISC1, SUF1), and components of both respiratory $(F M P$ s) and glycolytic metabolism (TDH1, PCK1). Specific roles for these proteins in low oxygen responses has long been recognized $O L E 1$, for example, is a canonical hypoxia response gene - but these data confirm that they are a widespread source of transcriptional regulation by oxygen. We propose that yeast has evolved lipid homeostasis machinery to adjust enzyme expression levels in accordance to environmental conditions, but has then adapted these sensors to act upon a wide range of molecular functions that are associated with these conditions. For several genes, including FLO1, sensing of unsaturated lipids is the dominant pathway for oxygen-induced activation, as transcript levels upon OLE1 repression or p90 induction closely mimic those upon hypoxia. However, for most targets it is likely that SPT23/ $M G A 2$ serve only a part of a complex response from several oxygen sensors in the cell. Ergosterol synthesis is also oxygen dependent, and Sterol Regulatory Element Binding Proteins (SREBP) have been identified as regulating hypoxia adaptation in fission yeast (Hughes et al., 2005) as well as two families (DAN and TIR) of hypoxic response genes in S. cerevisiae (Davies and Rine, 2006). The emerging roles for different lipid sensors in the yeast hypoxic response can therefore explain the varying timescales and oxygen concentration regimes noted for the induction of different hypoxic response genes, which cannot be explained by the classic yeast oxygen response regulators, Hap1p and Rox1p, as they are singularly activated by heme biosynthesis (Kwast et al., 1998). In metazoans, hypoxia-inducible factor (HIF-1) acts as a master regulator of hypoxic genes, and along with and its associated hydroxylases is thought to act as a direct sensor to changes in oxygen concentration (Semenza, 2004). In contrast, yeast relies on secondary messengers such as unsaturated fatty acids whose synthesis is intrinsically regulated by environmental oxygen concentration. One limitation of such a sensing mechanism is its temporal response: activation remains until membrane composition is diluted by new lipid synthesis, and is therefore unaffected by acute or short-term changes in the environment. Such kinetics could also be beneficial, however, by allowing the cell to integrate long-term environmental stimuli before committing on major changes to its physiology, such as rewiring basic metabolism or engagement in primitive collective behaviors like flocculation.

\section{Conclusion}

Here we introduced a metabolic engineering approach for investigating endogenous lipid synthesis pathways. Transcriptional modulation of the lipid desaturase gene $O L E 1$ generated a budding yeast strain in which lipid unsaturation and membrane fluidity is under experimental control. Work with this strain led to the unexpected discovery of a non-canonical flocculation pathway, in which ER-localized membrane ordering sensors trigger expression of FLO1. This pathway is triggered by low-oxygen conditions in wild-type cells, and provides a mechanistic explanation for sedimentation phenotypes important in industrial yeast. We show that the FLO1 pathway is part of a larger transcriptional response to low oxygen that is mediated by lipid unsaturation and is activated during anaerobic fermentation. 


\section{Supplemental material}

The online supplemental material includes 7 figures, 3 tables, and detailed methods for plasmid and strain construction. Fig. S1 shows the screening of different OLE1 promoter substitutions. Fig. S2 shows the effect of $O L E 1$ repression on exponential growth and cell-cell aggregation. Fig. S3 dissects the flocculation phenotype under OLE1 repression and shows in particular the influence of membrane fluidizing media supplements on yeast flocculation. Fig. S4 shows control measurements of membrane lipid composition upon indicated conditions (gene expression, gene knockouts, media supplementation). Fig. S5 shows controls of membrane fluidity induced processing of ${ }^{\mathrm{myc}} \mathrm{Spt} 23$ and ${ }^{\mathrm{myc}} \mathrm{Mga} 2 \mathrm{p}$ and reveals that intracellular Spt23p levels have no impact on proteolytic processing and release of ${ }^{\mathrm{myc}} \mathrm{Spt} 23 \mathrm{p} 90$. Fig. S6 demonstrates that acute hypoxia is not sufficient for changes in lipid unsaturation and FLO1 expression. Fig. S7 shows results of global expression analysis of membrane fluidity genes under long-term hypoxia. Tables $\mathrm{S} 1$ and $\mathrm{S} 2$ list all plasmids and primers used in this study. Table S3 shows functional groups of genes significantly upregulated by $O L E 1$ repression and significantly upregulated by $S P T 23 p 90$ or $M G A 2 p 90$ expression.

\section{Acknowledgments}

D.D. and I.B. performed the experiments. T.dR. carried out bioinformatics. D.D., T.dR, A.B, J.D.K. and I.B. discussed the results and wrote the manuscript. This work was supported by Junior Fellowship from the Miller Institute for Basic Research to I.B., by National Science Foundation grants MCB-1330914 and MCB-1341894 to J.D.K., by the Joint BioEnergy Institute, which is funded by the U.S. Department of Energy, Office of Science, Office of Biological and Environmental Research, under Contract DEAC02-05CH11231, and by a grant from the Deutsche Forschungsgemeinschaft (BE1181/10-1) to A.B. The authors thank Vanessa Kohl, Suzan Yilmaz, Charles Denby, and Dan Arlow for experimental assistance and helpful discussions.

\section{References}

van Meer, G., Voelker, D.R., Feigenson, G.W., 2008. Membrane lipids: where they are and how they behave. Nat. Rev. Mol. Cell Biol. 9, 112-124.

Klose, C., Surma, M.A., Gerl, M.J., Meyenhofer, F., Shevchenko, A., Simons, K., 2012. Flexibility of a eukaryotic lipidome-insights from yeast lipidomics. PLoS One 7, e35063.

Bossie, M.A., Martin, C.E., 1989. Nutritional regulation of yeast delta-9 fatty acid desaturase activity. J. Bacteriol. 171, 6409-6413.

Zidovetzki, R., Levitan, I., 2007. Use of cyclodextrins to manipulate plasma membrane cholesterol content: evidence, misconceptions and control strategies. Biochim. Biophys. Acta 1768, 1311-1324.

Spector, A.A., Yorek, M.A., 1985. Membrane lipid composition and cellular function. J. Lipid Res. 26, 1015-1035.

Hazel, J.R., 1995. Thermal adaptation in biological membranes: is homeoviscous adaptation the explanation? Annu. Rev. Physiol. 57, 19-42.

Ingram, L.O., 1976. Adaptation of membrane lipids to alcohols. J. Bacteriol. 125, 670-678.

Weijers, R.N., 2012. Lipid composition of cell membranes and its relevance in type 2 diabetes mellitus. Curr. Diabetes Rev. 8, 390-400.

Igal, R.A., 2010. Stearoyl-CoA desaturase-1: a novel key player in the mechanisms of cell proliferation, programmed cell death and transformation to cancer. Carcinogenesis 31, 1509-1515.

Hashimoto, K., Yoshizawa, A.C., Okuda, S., Kuma, K., Goto, S., Kanehisa, M., 2008. The repertoire of desaturases and elongases reveals fatty acid variations in 56 eukaryotic genomes. J. Lipid Res 49, 183-191.

Barelli, H., Antonny, B., 2016. Lipid unsaturation and organelle dynamics. Curr. Opin. Cell Biol. 41, 25-32.

Stukey, J.E., McDonough, V.M., Martin, C.E., 1989. Isolation and characterization of OLE1, a gene affecting fatty acid desaturation from Saccharomyces cerevisiae. J. Biol. Chem. 264, 16537-16544.
Casanovas, A., Sprenger, R.R., Tarasov, K., Ruckerbauer, D.E., Hannibal-Bach, H.K., Zanghellini, J., Jensen, O.N., Ejsing, C.S., 2015. Quantitative analysis of proteome and lipidome dynamics reveals functional regulation of global lipid metabolism. Chem. Biol. 22, 412-425.

Choi, J.Y., Stukey, J., Hwang, S.Y., Martin, C.E., 1996. Regulatory elements that control transcription activation and unsaturated fatty acid-mediated repression of the Saccharomyces cerevisiae OLE1 gene. J. Biol. Chem. 271, 3581-3589.

Hoppe, T., Matuschewski, K., Rape, M., Schlenker, S., Ulrich, H.D., Jentsch, S., 2000. Activation of a membrane-bound transcription factor by regulated ubiquitin/ proteasome-dependent processing. Cell 102, 577-586.

Zhang, S., Burkett, T.J., Yamashita, I., Garfinkel, D.J., 1997. Genetic redundancy between SPT23 and MGA2: regulators of Ty-induced mutations and Ty1 transcription in Saccharomyces cerevisiae. Mol. Cell Biol. 17, 4718-4729.

Rape, M., Hoppe, T., Gorr, I., Kalocay, M., Richly, H., Jentsch, S., 2001. Mobilization of processed, membrane-tethered SPT23 transcription factor by CDC48(UFD1/NPL4), a ubiquitin-selective chaperone. Cell 107, 667-677.

Burkett, T.J., Garfinkel, D.J., 1994. Molecular characterization of the SPT23 gene: a dosage-dependent suppressor of Ty-induced promoter mutations from Saccharomyces cerevisiae. Yeast 10, 81-92.

Dula, M.L., Holmes, S.G., 2000. MGA2 and SPT23 are modifiers of transcriptional silencing in yeast. Genetics 156, 933-941.

Covino, R., Ballweg, S., Stordeur, C., Michaelis, J.B., Puth, K., Wernig, F., Bahrami, A., Ernst, A.M., Hummer, G., Ernst, R., 2016. A eukaryotic sensor for membrane lipid saturation. Mol. Cell 63, 49-59.

Brachmann, C.B., Davies, A., Cost, G.J., Caputo, E., Li, J., Hieter, P., Boeke, J.D., 1998. Designer deletion strains derived from Saccharomyces cerevisiae S288C: a useful set of strains and plasmids for PCR-mediated gene disruption and other applications. Yeast 14, 115-132.

Bligh, E.G., Dyer, W.J., 1959. A rapid method of total lipid extraction and purification. Can. J. Biochem. Physiol. 37, 911-917.

Printen, J.A., Sprague, G.F., Jr., 1994. Protein-protein interactions in the yeast pheromone response pathway: ste5p interacts with all members of the MAP kinase cascade. Genetics 138, 609-619.

Van Mulders, S.E., Christianen, E., Saerens, S.M., Daenen, L., Verbelen, P.J., Willaert, R., Verstrepen, K.J., Delvaux, F.R., 2009. Phenotypic diversity of Flo protein familymediated adhesion in Saccharomyces cerevisiae. FEMS Yeast Res. 9, 178-190.

Collart, M.A., Oliviero, S., 2001. Preparation of yeast RNA. Curr. Protoc. Mol. Biol., (Chapter 13, Unit13 12).

Mao, X., Hu, Y., Liang, C., Lu, C., 2002. MET3 promoter: a tightly regulated promoter and its application in construction of conditional lethal strain. Curr. Microbiol. 45, $37-40$.

Asadollahi, M.A., Maury, J., Moller, K., Nielsen, K.F., Schalk, M., Clark, A., Nielsen, J., 2008. Production of plant sesquiterpenes in Saccharomyces cerevisiae: effect of ERG9 repression on sesquiterpene biosynthesis. Biotechnol. Bioeng. 99, 666-677.

Paradise, E.M., Kirby, J., Chan, R., Keasling, J.D., 2008. Redirection of flux through the FPP branch-point in Saccharomyces cerevisiae by down-regulating squalene synthase. Biotechnol. Bioeng. 100, 371-378.

Lentz, B.R., 1989. Membrane "fluidity" as detected by diphenylhexatriene probes. Chem. Phys. Lipids 50, 171-190.

Heipieper, H.J., Isken, S., Saliola, M., 2000. Ethanol tolerance and membrane fatty acid adaptation in adh multiple and null mutants of Kluyveromyces lactis. Res. Microbiol. $151,777-784$.

Stratford, M., 1992a. Lectin-mediated aggregation of yeasts-yeast flocculation. Biotechnol. Genet. Eng. Rev. 10, 283-341.

Guinard, J., Lewis, J.M., 1993. Study of the phenomenon of agglomeration in the yeast Saccharomyces cerevisiae. J. Inst. Brew. 99, 487-503.

Stratford, M., Assinder, S., 1991. Yeast flocculation: Flo1 and NewFlo phenotypes and receptor structure. Yeast 7, 559-574.

Mukhopadhyay, K., Kohli, A., Prasad, R., 2002. Drug susceptibilities of yeast cells are affected by membrane lipid composition. Antimicrob. Agents Chemother. 46, 3695-3705.

Sinicrope, F.A., Dudeja, P.K., Bissonnette, B.M., Safa, A.R., Brasitus, T.A., 1992. Modulation of P-glycoprotein-mediated drug transport by alterations in lipid fluidity of rat liver canalicular membrane vesicles. J. Biol. Chem. 267, 24995-25002.

Liu, H., Styles, C.A., Fink, G.R., 1996. Saccharomyces cerevisiae S288C has a mutation in FLO8, a gene required for filamentous growth. Genetics 144, 967-978.

Jiang, Y., Vasconcelles, M.J., Wretzel, S., Light, A., Gilooly, L., McDaid, K., Oh, C.S., Martin, C.E., Goldberg, M.A., 2002. Mga2p processing by hypoxia and unsaturated fatty acids in Saccharomyces cerevisiae: impact on LORE-dependent gene expression. Eukaryot. Cell 1, 481-490.

Nakagawa, Y., Sakumoto, N., Kaneko, Y., Harashima, S., 2002. Mga2p is a putative sensor for low temperature and oxygen to induce OLE1 transcription in Saccharomyces cerevisiae. Biochem Biophys. Res. Commun. 291, 707-713.

Kandasamy, P., Vemula, M., Oh, C.S., Chellappa, R., Martin, C.E., 2004. Regulation of unsaturated fatty acid biosynthesis in Saccharomyces: the endoplasmic reticulum membrane protein, Mga2p, a transcription activator of the OLE1 gene, regulates the stability of the OLE1 mRNA through exosome-mediated mechanisms. J. Biol. Chem. $279,36586-36592$.

Auld, K.L., Brown, C.R., Casolari, J.M., Komili, S., Silver, P.A., 2006. Genomic association of the proteasome demonstrates overlapping gene regulatory activity with transcription factor substrates. Mol. Cell 21, 861-871.

Andreasen, A.A., Stier, T.J., 1954. Anaerobic nutrition of Saccharomyces cerevisiae. II. Unsaturated fatty acid requirement for growth in a defined medium. J. Cell. Comp. Physiol. 43, 271-281.

Kwast, K.E., Burke, P.V., Poyton, R.O., 1998. Oxygen sensing and the transcriptional regulation of oxygen-responsive genes in yeast. J. Exp. Biol. 201, 1177-1195. 
Herschlag, D., Johnson, F.B., 1993. Synergism in transcriptional activation: a kinetic view.. Genes Dev. 7, 173-179.

Straver, M.H., vd Aar, P.C., Smit, G., Kijne, J.W., 1993. Determinants of flocculence of brewer's yeast during fermentation in wort. Yeast 9, 527-532.

Soares, E.V., Teixeira, J.A., Mota, M., 1994. Effect of cultural and nutritional conditions on the control of flocculation expression in Saccharomyces cerevisiae. Can. J. Microbiol. 40, 851-857.

Smit, G., Straver, M.H., Lugtenberg, B.J., Kijne, J.W., 1992. Flocculence of Saccharomyces cerevisiae cells is induced by nutrient limitation, with cell surface hydrophobicity as a major determinant. Appl. Environ. Microbiol. 58, 3709-3714.

Stratford, M., 1992b. Yeast flocculation: a new perspective. Adv. Micro. Physiol. 33, $2-71$.

Soares, E.V., Mota, M., 1996. Flocculation onset, growth phase, and genealogical age in Saccharomyces cerevisiae. Can. J. Microbiol 42, 539-547.

Powell, C.D., Quain, D.E., Smart, K.A., 2003. The impact of brewing yeast cell age on fermentation performance, attenuation and flocculation. FEMS Yeast Res 3, $149-157$.

Barker, M.G., Smart, K.A., 1996. Morphological changes associated with the cellular aging of a brewing yeast strain. J. Am. Soc. Brew. Chem. 54, 121-126.

Verstrepen, K.J., Derdelinckx, G., Verachtert, H., Delvaux, F.R., 2003. Yeast flocculation: what brewers should know. Appl Microbiol Biotechnol. 61, 197-205.

Bester, M.C., Pretorius, I.S., Bauer, F.F., 2006. The regulation of Saccharomyces cerevisiae FLO gene expression and Ca2+-dependent flocculation by Flo8p and Mss11p. Curr. Genet 49, 375-383.

Govender, P., Domingo, J.L., Bester, M.C., Pretorius, I.S., Bauer, F.F., 2008. Controlled expression of the dominant flocculation genes FLO1, FLO5, and FLO11 in Saccharomyces cerevisiae. Appl. Environ. Microbiol. 74, 6041-6052.

Shen, H., Iha, H., Yaguchi, S., Tsurugi, K., 2006. The mechanism by which overexpression of Gts1p induces flocculation in a FLO8-inactive strain of the yeast Saccharomyces cerevisiae. FEMS Yeast Res. 6, 914-923.

Vasconcelles, M.J., Jiang, Y., McDaid, K., Gilooly, L., Wretzel, S., Porter, D.L., Martin, C.E., Goldberg, M.A., 2001. Identification and characterization of a low oxygen response element involved in the hypoxic induction of a family of Saccharomyces cerevisiae genes. Implications for the conservation of oxygen sensing in eukaryotes. J. Biol. Chem. 276, 14374-14384.

Kwast, K.E., Burke, P.V., Staahl, B.T., Poyton, R.O., 1999. Oxygen sensing in yeast: evidence for the involvement of the respiratory chain in regulating the transcription of a subset of hypoxic genes. Proc. Natl. Acad. Sci. U.S.A. 96, 5446-5451.

Holmberg, S.K.-B., M.C., 1978. A mutant of Saccharomyces cerevisiae temperature sensitive for flocculation. Influence of oxygen and respiratory deficiency on flocculence. Carlsberg Res. Commun. 43, 37-47.

Tesniere, C., Delobel, P., Pradal, M., Blondin, B., 2013. Impact of nutrient imbalance on wine alcoholic fermentations: nitrogen excess enhances yeast cell death in lipidlimited must. PLoS One 8, e61645.

Smukalla, S., Caldara, M., Pochet, N., Beauvais, A., Guadagnini, S., Yan, C., Vinces, M.D., Jansen, A., Prevost, M.C., Latge, J.P., et al., 2008. FLO1 is a variable green beard gene that drives biofilm-like cooperation in budding yeast. Cell 135, 726-737.

Iserentant, D., 1996. Practical aspects of yeast flocculation. Cerevisia 21, 30-33.

Steward, G.G., Russell, I., 1981. Yeast flocculation. Academic Press, New York.

Goossens, K.V., Ielasi, F.S., Nookaew, I., Stals, I., Alonso-Sarduy, L., Daenen, L., Van Mulders, S.E., Stassen, C., van Eijsden, R.G., Siewers, V., et al., 2015. Molecular mechanism of flocculation self-recognition in yeast and its role in mating and survival. MBio, 6.

Mortimer, R.K., Johnston, J.R., 1986. Genealogy of principal strains of the yeast genetic stock center. Genetics 113, 35-43.

Krishnamurthy, S., Plaine, A., Albert, J., Prasad, T., Prasad, R., Ernst, J.F., 2004. Dosagedependent functions of fatty acid desaturase Ole1p in growth and morphogenesis of Candida albicans. Microbiology 150, 1991-2003.

Oh, C.S., Martin, C.E., 2006. Candida albicans Spt23p controls the expression of the Ole1p Delta9 fatty acid desaturase and regulates unsaturated fatty acid biosynthesis. J. Biol. Chem. 281, 7030-7039.

Jiang, Y., Vasconcelles, M.J., Wretzel, S., Light, A., Martin, C.E., Goldberg, M.A., 2001. MGA2 is involved in the low-oxygen response element-dependent hypoxic induction of genes in Saccharomyces cerevisiae. Mol. Cell Biol. 21, 6161-6169.

Pagani, M.A., Casamayor, A., Serrano, R., Atrian, S., Arino, J., 2007. Disruption of iron homeostasis in Saccharomyces cerevisiae by high zinc levels: a genome-wide study. Mol. Microbiol. 65, 521-537.

Lyons, T.J., Villa, N.Y., Regalla, L.M., Kupchak, B.R., Vagstad, A., Eide, D.J., 2004. Metalloregulation of yeast membrane steroid receptor homologs. Proc. Natl. Acad. Sci. U.S.A. 101, 5506-5511.

Stewart, L.C., Yaffe, M.P., 1991. A role for unsaturated fatty acids in mitochondrial movement and inheritance. J. Cell Biol. 115, 1249-1257.

Hughes, A.L., Todd, B.L., Espenshade, P.J., 2005. SREBP pathway responds to sterols and functions as an oxygen sensor in fission yeast. Cell 120, 831-842.

Davies, B.S., Rine, J., 2006. A role for sterol levels in oxygen sensing in Saccharomyces cerevisiae. Genetics 174, 191-201.

Semenza, G.L., 2004. Hydroxylation of HIF-1: oxygen sensing at the molecular level. Physiology (Bethesda) 19, 176-182. 